\title{
Reply to letter to the editor concerning: Regulation of testicular descent
}

\author{
John M. Hutson ${ }^{1,2,3} \cdot$ Ruili $^{\mathrm{Li}^{1}} \cdot$ Bridget R. Southwell $^{1,3} \cdot$ Don Newgreen $^{1}$ • \\ Mary Cousinery ${ }^{1,3}$
}

Accepted: 20 April 2015/Published online: 16 May 2015

(C) Springer-Verlag Berlin Heidelberg 2015

Dr. Hadziselimovic reminds us that in evolution, the primary structure that descended is the caudal epididymis, and the testis appears to have descended secondarily. However, in the human, clearly descent of the testis is a major process occurring simultaneously with descent of the epididymis.

Dr. Hadziselimovic comments on whether the Wolffian duct development needs to be normal for testicular descent to occur, and our recent article does not address this, although this has been addressed with anatomical studies in the past, some of which he refers to in his list of references. $\mathrm{He}$ goes on to describe the relationship between androgen levels and epididymal development, as well as the likelihood that mutations in the FGF receptor 1 gene may be involved in cryptorchidism. He mentions the paper from 2010 describing boys with unilateral cryptorchidism where there is impaired expression of FGF receptor 1 in the undescended but not the descended testis. This suggests that expression of this gene may be impaired secondarily by maldescent, and it remains to be seen whether it is a primary cause for undescended testis itself.

This reply refers to the comment available at doi:10.1007/s00383-015-3714-z.

John M. Hutson

john.hutson@rch.org.au

1 F Douglas Stephens Surgical Research Group and Embryology Laboratory, Murdoch Childrens Research Laboratory, Melbourne, Australia

2 Urology Department, The Royal Children's Hospital, Parkville, 3052, Melbourne, VIC, Australia

3 Department of Paediatrics, University of Melbourne, Melbourne, Australia
In the rest of his letter, Dr. Hadziselimovic comments on the role of the gubernaculum, and whether it is the proximal or the distal portion of the gubernaculum which is most important. This was not an issue that I addressed directly in this short review mostly because of limitation of space. In the past we have written extensively on gubernaculum and the different parts of this structure and their role in testicular descent.

Finally, Dr. Hadziselimovic comments on the role of the various hormones anti-müllerian hormone insulin-like factor 3 and testosterone. This leads to the fact that there is some discrepancy between human examples of anti-müllerian hormone dysfunction in persistent müllerian duct syndrome versus what is found in mouse models. The discrepancy between mouse models and the human with persistent müllerian duct syndrome remains unresolved, and will clearly be the subject of further research.

We thank Dr. Hadziselimovic for his interest in our manuscript. 